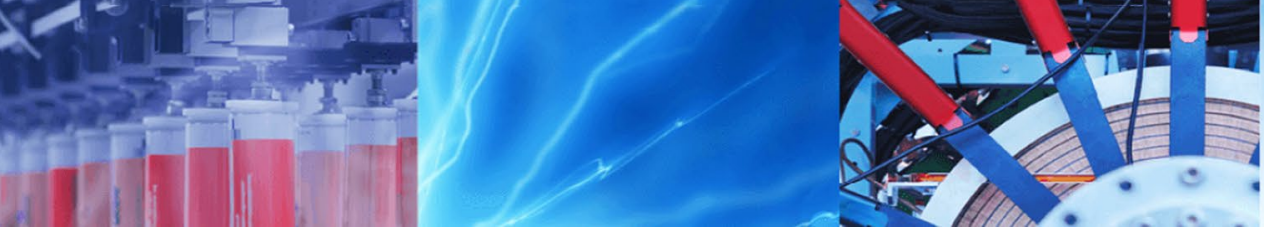

Review Paper

\title{
An overview of numerical methods for the first kind Fredholm integral equation
}

\author{
Di Yuan ${ }^{1,2} \cdot$ Xinming Zhang $^{1}$ \\ () Springer Nature Switzerland AG 2019
}

\begin{abstract}
In the field of engineering technology, many problems can be transformed into the first kind Fredholm integral equation, which has a prominent feature called "ill-posedness". This property makes it difficult to find the analytical solution of first kind Fredholm integral equation. Therefore, how to find the numerical solution of first kind Fredholm integral equation has been a common concern of domestic and overseas scholars in recent years. In this article, various numerical solution methods of first kind Fredholm integral equation are introduced in detail. First, the existence and convergence of the solution of the integral equation are given. Second, the current mainstream numerical methods, such as regularization method, wavelet analysis method and multilevel iteration method are introduced in detail. Finally, we presented a concise overview of the numerical method of first kind Fredholm integral equation.
\end{abstract}

Keywords First kind Fredholm integral equation · III-posedness · Numerical solution · Convergence

\section{Introduction}

Mathematics and computer science are widely used to solve complex problems in different fields. In engineering, physics, mechanics, atmospheric science, geological exploration, remote sensing, non-destructive testing, medical imaging, military science and other fields, a large number of scientific and engineering problems can be reduced to solving the first kind of Fredholm integral equation [1-4]. Further more, several mathematical problems, such as the problem of harmonic continuation, the backwards heat equation, numerical inversion of the Laplace transform and numerical differentiation, can be formulated in the form of these equations [5-9]. In addition, the initial and boundary value problems of differential equations can also be transformed into integral equations to solve, the advantage of which is that only the integral equation needs to be solved. The initial and boundary value problems of differential equations are solved discretely along the boundary.
However, the smoother the kernel of the first kind of Fredholm integral equation is, the more unstable it is. As we all know, the most prominent characteristic of the first kind of Fredholm integral equation is its "ill-posedness". This property makes it difficult to solve. Only a few integral equations can find their analytical solutions. A large number of integral equations need to find their numerical solutions. Therefore, to study the effective numerical solution of this kind of integral equation has become a research direction that mathematicians, natural science workers, and engineering technicians strive to open up. In recent years, the numerical solution of Fredholm integral equation has been greatly developed [10-14].

Based on the research on the numerical solutions of the first kind of Fredholm integral equation by scholars nationally and abroad, this paper gives a concise summary of the numerical solutions. It can be divided into the following parts: the first section mainly gives the research background of the first kind of Fredholm integral equation; the second section mainly gives the basic concepts,

Di Yuan, dyuanhit@gmail.com | ${ }^{1}$ School of Science, Harbin Institute of Technology, Shenzhen, China. ${ }^{2}$ School of Computer Science, Harbin Institute of Technology, Shenzhen, China.

SN Applied Sciences (2019) 1:1178 | https://doi.org/10.1007/s42452-019-1228-3

Received: 22 May 2019 / Accepted: 3 September 2019 / Published online: 7 September 2019 
definitions, and theorems of the solution of the first kind of Fredholm integral equation, and gives the convergence criteria and the accuracy criteria of the solution of the first kind of Fredholm integral equation; the third section mainly gives the existing first kind of Fredholm integral equation. The numerical solution of the equation is summarized, including direct numerical integration method, regularization method, wavelet analysis method, GMRES method, multi-layer iterative algorithm, CAS wavelet solution, multiple constraints smoothing method, improved Tikhonov regularization method, etc. The fourth section summarizes the existing methods of solving the numerical solution of the first kind of Fredholm integral equation, taking into account the objective. Based on the advantages of the former popular intelligent algorithm, the basic idea of solving the numerical solution of the first kind of Fredholm integral equation by combining the intelligent algorithm is proposed.

\section{Analysis of the existence and convergence of solutions to integral equations}

Firstly, we give the general form of the first kind Fredholm integral equation:

$\int_{b}^{a} k(x, t) g(t) d t=f(x)$

where $k(x, t)$ and $f(x)$ are known functions, $a$ and $b$ denotes upper and lower bound constant of integration, $k(x, t)$ is usually called the kernel function of the integral equation, which determines the basic properties of the integral equation. $f(x)$ is called the free term of the integral equation and $g(x)$ is the function to be solved. The existence and convergence of solutions of the integral equation and the current research situation are given below, which can provide a basis for determining whether the solution of the integral equation can be solved and whether the solution obtained has convergence.

\subsection{The existence of solutions and the basic theorem of convergence}

The existence and convergence of solutions of integral equations are the fundamental basis for their solvability. Firstly, the basic criterion theorem for the existence and convergence of solutions is given.

Theorem 2.1 [15] If $\lambda_{i}$ and $\varphi_{i}(x), \phi_{i}(x)$ are the sequence of eigenvalues of the first kind Fredholm integral equation 1 , and the orthogonal standard system $\varphi_{i}(x)$ is complete. Then the necessary and sufficient condition for the first kind
Fredholm integral equation to have a solution is: the series $\sum_{i=1}^{+\infty} \lambda_{i}^{2}\left|f_{i}\right|^{2}$ converges, where $f_{i}=\left(f, \varphi_{i}\right)$.

Theorem 2.2 [15] When the first kind of integral equation has a solution, (i) if and only if the orthogonal standard function system $\varphi_{i}(x)$ is complete for $f(x)$, the sequence $K g_{n}$ uniformly converges to $f(x)$; (ii) if and only if the orthogonal standard function system $\phi_{i}(x)$ is complete for $g(x)$, the sequence $g_{n}(x)$ uniformly converges to $g(x)$.

Theorem 2.3 [16] Suppose that the solution $g$ of integral equation $f(x)$ exists and satisfies the condition $g_{0}=K^{*} h_{0}, h_{0} \in L_{2}[a, b]$. If and only if $\phi_{i}$ is complete about $g, g_{n}$ converges to $g$ on average. If $g=K^{*} h, h \in L_{2}[a, b]$ is satisfied, $g_{n}$ converges to $g$ on average.

Through the above discriminant theorem, we can basically determine whether an integral equation has a solution and whether the solution obtained is convergent. Then, the present research status of the existence and convergence of solutions of integral equations by scholars is given.

\subsection{The research status of the existence and convergence of solutions}

With the development of science and technology, the problem of solving an integral equation becomes more and more important. Many scholars have studied the existence and convergence of numerical solutions of the first kind of Fredholm integral equation.

In the study of the existence and convergence of numerical solutions of integral equation, Yun [17] gives the representation theorem and iteration theorem of the first kind of Fredholm integral equation with $L_{2}$ integral kernels which can be expressed in the form of $A x \doteq y$, and verifies the reliability of the theorem based on empirical analysis. Groetsch [5] proposes an asymptotic convergence analysis for the first kind of Fredholm integral equation with simple degenerate kernels for Tikhonov functional based on orthogonal variations. The convergence theorem proves that the mean square, uniform norm, and Tikhonov regularization methods are valid under any norm when the data are inaccurate. Li and Zhao [18] made a brief analysis of the reliability of the numerical solution of the first kind of integral equation in Fabry-Perot interferometric inversion spectroscopy. It was proved that when $\Delta e=2 / x, \Delta x=2 / e$ and the equidistant sampling points were an appropriate odd number, the integral equation could be discretized into a stable linear equation group by using the simplest rectangular quadrature formula, but the solution of the equation group was not the solution of the original integral equation. Rajan [7] considers an 
improved convergence analysis based on Hilbert space and uses the Tikhonov regularization method to solve the first kind of Fredholm integral equation. The same results are obtained for the general equation and Groetsch equation. Maleknejad et al. [8] consider the first kind of Fredholm integral equation with bounded inverse degenerate kernels. The convergence of the numerical method is proved by combining the wavelet method with the collocation method to solve the equation. The conjugate gradient method is used to solve the discretized integral equation of linear equations, which verifies the practicability of the numerical method. Hong and Cui [19] discussed the stability of the exact solutions of the first kind of Fredholm integral equation in reproducing kernel space, and verified that the first kind of Fredholm integral equation with reproducing kernel space is a well-posed problem. The numerical experiments show the effectiveness of the algorithm. Zhang and Wang [20] studied the existence of positive solutions of the Fredholm type integral equation $u(t)=\int_{0}^{1} k(t, s) f(s, u(s)) d s$ by using the fixed point index theorem on cone. The results show that as long as the growth rate of the non-linear term is appropriate, there exists a positive solution to the problem. Liu [21] discussed the direct discretization method for a class of Fredholm integral equation of the first kind with a unique solution and gave the error estimates for solving this kind of integral equation by using different discretization methods theoretically. The error bounds for the perturbation of the right-hand term of the equation are given. The numerical experiments show that the Gauss method has a good effect under certain conditions. Han Hood et al. [22] studied the existence and uniqueness of solutions of the first kind of Fredholm integral equations generated by the Dirichlet problem of Laplace equation in two-dimensional multi-connected domain; introduced a discriminant index $\gamma_{N} ;$ proved that the necessary and sufficient condition for the existence and uniqueness of solutions of the first kind of Fredholm integral equations is $\gamma_{N} \neq 0$; and verified the theoretical results by numerical examples.

\section{The first kind of Fredholm integral equation numerical method}

For the first kind of Fredholm integral equation, the ill-posedness leads to its solvability which is different from other integral equations. Generally, the existence of solutions of the first kind of Fredholm integral equation usually has infinite or infinite solutions, which hinders the progress of scientific research in both engineering and image processing fields. So it is very important to find the numerical solution of the first kind of Fredholm integral equation. Many scholars have studied the numerical solution of the integral equation from different aspects, and achieved ideal results [23-26].

\subsection{Regularization method}

Regularization method is the most common method for solving the first kind of Fredholm integral equation [12, 27-31]. From the existing research, the Tikhonov regularization method is an effective method for solving ill-posed problems [32]. The basic idea is to use the prior information of the solution to transform the original problem into an appropriate and well-posed optimization problem to obtain a regular solution, and then to obtain a stable method to solve the original problem. In recent years, many researchers have solved the first kind of Fredholm integral equation from different regularization operators and obtained more perfect conclusions.

Lee and Prenter [27] solved the numerical solution of the first kind of Fredholm integral equation by means of finite rank and replacing $T x=y$ with $T_{n} x=y_{n}, n=1,2, \ldots$ $\left(T, T_{n}\right.$ can be regarded as an operator from $L_{2}[a, b]$ to $L_{2}[c, d]$ or from $L_{\infty}[a, b]$ to $\left.L_{\infty}[c, d]\right)$. The discrete or continuous integrity analysis of $T x=y$ problem is given. By means of the least square norm, the comparison between the solutions of the discrete problem $T_{n} x=y_{n}$ and the continuous problem $T x=y$ is given, and the convergence analysis of the discrete problem is given. Perminov [29] gives the numerical solution of the linear two-dimensional Fredholm integral equation of the first kind by Tikhonov regularization method. The simplicity and effectiveness of the algorithm are verified by an example. When You and Miao [30] discussed the solution of the first kind of Fredholm integral equation with complex kernels, the extension of the Tikhonov regularization method was given, and the convergence of approximate regularization method was discussed. The practicability of the optimal regularization parameter is verified based on the two-dimensional wave equation. Tanana et al. [33] propose a variational regularization method with a regularization parameter from the residual principle and reducing the problem to a system of linear algebraic equations. The accuracy of the approximate solution is estimated with allowance for the error of the finite dimensional approximation of the problem. The use of this approach is illustrated by solving an inverse boundary value problem for the heat conduction equation. Caldwell [34] validates two numerical methods, direct orthogonal method, and boundary integral method, to solve the first kind of Fredholm integral equation. Aiming at the core of the integral equation, the results of the first type of ill-posed equation and the second type of wellposed equation are compared by regularization method, and the errors generated by comparison verify the feasibility of the algorithm in solving the first type of integral 
equation. Pereverzeva [35] uses a projection method to estimate errors in solving the first kind of Fredholm integral equation $A x=y+\xi$ with random perturbations. Under the assumption that integral operator $A$ has a differentiable kernel $(\phi, \beta)$ and the mathematical expectation of $\|\xi\|^{2}$ does not exceed $\sigma^{2}$, a more complete inference is obtained than that of Vainikko and Plato in EEE results. Dobner [36] studied the numerical method of the first kind of linear Fredholm integral equation. Based on the series expansion technique, the kernel of the integral equation is expanded into the degenerate kernel part of finite dimension and the minimal norm part of infinite dimension. Without considering the influence of the remainder, the original equation can be transformed into a degenerate nuclear problem. A regularization method is given and a numerical approximation method is obtained by strictly controlling rounding errors. Li et al. [37-39] proposed a new optimization regularization algorithm based on the general regularization method for solving the first kind of Fredholm integral equation. In this paper, an improved Tikhonov regularization strategy for solving general operator equations is proposed by using the idea of constructing regularizers: $x_{r}^{\alpha, \delta}=R_{r}^{\alpha} y^{\delta}=\left(\alpha I+\left(K^{*} K\right)^{r / 2}\right)^{-2 / r} K^{*} y^{\delta}$. By priority selecting the regular parameters, it is proved that the regular solution can obtain higher convergence efficiency. A kind of Fredholm integral equation is solved numerically by means of the software of Matlab. The results show that the improved Tikhonov regularization is more accurate than the usual Tikhonov regularization, and the regularization solution can achieve a convergence rate which is basically consistent with the theoretical analysis. Considering the great difficulty in solving the discrete system of the first kind of Fredholm integral equation. Maleknejad et al. [40] reduced the dimension of discrete linear equations of the integral equation by using wavelet basis and collocation method. Then the conjugate gradient method is used to solve the algebraic equations and a method to estimate the error bound is given. The dimension of the discrete system is effectively reduced, and the solving process is improved. When the data of the first Fredholm equation satisfy some spectral conditions, Dan and Shklyar [41] verifies the weak convergence and norm convergence of the sequence generated by Cimmino algorithm (an iterative projection method for finding an almost common measurable family of points in Hilbert space of closed convex sets). Based on this, a criterion for the existence of solutions is obtained when solving this kind of equation. If the equation has solutions, the Cimmino algorithm can generate gauge approximation solutions.

Nair and Pereverzev [42] consider a collocation method to solve the first kind of Fredholm integral equation. The collocation matrix obtains the minimum singular value in a priori manner. In this case, the collocation value is used as a regularization parameter. If the prior information is not available, the regularization parameters are selected by matching the Tikhonov regularization method. Generally, collocation normalization is considered when the smoothing solution is the source condition of the monotone exponential function. It covers all types of research on smooth Tikhonov regularization theory. A posteriori regularization parameter is selected to achieve the accuracy of an optimal deterministic noise model without any smooth solution. For the collocation method to study the numerical solution of the first kind of Fredholm integral equation, its accuracy and computational complexity mainly depend on the selected basis function. Up to now, the basis functions used most by collocation method include trigonometric function, spline function, B-spline function, Legendre wavelet function [43] and so on. These methods can achieve a higher order accuracy under certain conditions. However, the accuracy of these numerical methods is generally related to $\alpha$. When $\alpha \rightarrow 1$, the accuracy will generally be lower and lower until the order of 0 . Zeng et al. [44] proposed a collocation method using Lagrange interpolation polynomial as the basis function. The collocation method successfully dealt with the weak singularity of the integral equation by using the partial integral formula, and the accuracy involved was independent of $\alpha$.

When Rajan [45] solves the convergence analysis of the first kind of Fredholm integral equation, he proposes to change the rule parameter selection strategy based on the prior parameter to the rule parameter selection strategy based on the posterior parameter and proposes a kind of deviation principle according to Hilbert space characteristics and numerical analysis. Wazwaz [46] uses regularization method and successive approximation method to deal with ill-posed problems when solving the first kind of Fredholm integral equation. The reliability of the regularization method is verified by an example. Micheli and Viano [47] considered the ill-posed problem of the classical Fredholm integral equation of the first kind given by Hadamard. If the integral operator is self-adjoint and gives a set of eigenfunctions, the general solution of the integral equation can be written in the form of eigenfunction expansion. One of the regularization methods is to combine the truncation idea of compact $\varepsilon$ coverage consideration method and give the allowable phase error of the expansion by the global prior method. Yin [48] proposed a discrete regularization algorithm based on matrix singular value decomposition. By choosing appropriate regularization parameters (using a prior selection of regularization parameters, deviation principle or absorption compatibility principle to select regularization parameters), the solution method of the first kind of Fredholm integral equation is given. Yang [49] studied the theoretical analysis of the first kind of Fredholm inverse problem 
on infinite intervals, solved the problem by regularization method, constructed the flattening function by using Sobolev space norm, and then obtained the regularization operator, and determined the regularization parameters according to Morozov deviation principle. The existence and uniqueness of regularization solutions are proved by constructing the minimization sequence of flattening functional, which lays a foundation for the numerical solution of the infinite Fredholm problem of the first kind. Min et al. [50,51] used numerical integral formula to discretize the solution of the first kind of three-dimensional Fredholm integral equation. By introducing the regularization method, the discrete integral equation was transformed into a well-posed problem, and its numerical solution was obtained by the generalized minimal residual algorithm. The method is simple and effective. When solving the first kind of weak singular Kernel Fredholm integral equation, the equation is transformed by Cress transformation, and the transformed equation is discretized by Gauss-Legendre method. A discrete system of ill-posed linear equations is obtained, which is solved numerically by combining the regularization method. The feasibility and effectiveness of the proposed method are verified by numerical simulation.

Lin and Yang [52] proposed a new regularization method based on weighted $H^{1}$ seminorm. The efficiency of the method is verified by the examples of a onedimensional and two-dimensional integral equation. $\mathrm{Wu}$ [53] solves the first kind of Fredholm integral equation of one dimension with a discontinuous true solution by total variation regularization and then solves the first kind of Fredholm integral equation of two dimensions discretely. The discretization and singular value decomposition of the first kind of two-dimensional Fredholm integral equation are described systematically. In order to obtain a stable numerical solution, the integral kernels are discretized and solved by the regularization method. Combined with the structure of the coefficient matrix, the L-curve method and deviation principle are combined with regularization method and solved. It is verified that the solution of the first kind of two-dimensional Fredholm integral equation with nonsingular kernels depends on the variation of the kernels and the smoothness of the true solution, and the perturbation of the data and the mesh subdivision affect the accuracy of the numerical solution. Zhang [54] introduced the discretization and regularization theory of ill-posed problems, and several kinds of continuous regularization methods: singular value decomposition, Landweber and Fridman iteration methods. These theories are demonstrated by the compaction theory. The method of Sidi formula interpolation is proposed to approximate the solution of an integral equation. Two projection methods, Galerkin method, and least square method, are given as regularization strategies. After the integral equation is discretized, the discrete solution is regularized, and its convergence and numerical stability are proved. Two Tikhonov regularization methods, the quasi-solution method, and the minimum norm solution method, are proposed. The results obtained by the minimum norm solution method are relatively stable through an example. A new Tikhonov regularization matrix is constructed. The generalized cross-check method (GCV) is used to select regularization parameters. Compared with the traditional Tikhonov regularization method and truncated singular value decomposition method, numerical examples show that the error of this method is small. Mao [55] applies the Tikhonov regularization method to the numerical solution of the first kind of Fredholm integral equation and uses different strategies to select regularization parameters to obtain better numerical fitting results. Furthermore, three kinds of smooth functions, continuously differentiable, continuous non-differentiable and discontinuous functions, are selected to illustrate the effectiveness of the algorithm. The numerical solution of two Fredholm integral equations of the first kind derived from the field of geological exploration is used to verify the algorithm.

\subsection{Wavelet method}

As early as 1991, wavelet was used to solve the numerical solution of the integral equation. In recent years, different wavelet functions have been applied to solve the first kind of Fredholm integral equation, such as CAS wavelet, Legendre wavelet, Coifnan wavelet, Haar wavelet and so on, which have produced relatively ideal results.

Yousefi and Banifatemi [56] proposed a numerical method based on CAS wavelet approximation for solving the first kind of Fredholm integral equation. Firstly, the properties of CAS wavelet are given, and the solution of the first kind of Fredholm integral equation is transformed into the solution of algebraic equations by using CAS wavelet approximation method. The efficiency of the algorithm is verified by numerical examples. Han et al. [57-59] also started from CAS wavelet, transformed the integral equation into algebraic equation group by using CAS wavelet collocation method, and then solved the algebraic equation group by Tikhonov regularization method. Legendre multi-wavelet collocation method is proposed to solve the first kind of Fredholm integral equation. The integral equation is transformed into general algebraic equations by using wavelet functions as a group of bases. The integral equations are transformed into linear equations by Legendre wavelet Galerkin method. For $n+1$ different regularizers, Tikhonov regularization method is used to solve them, and different stable solutions of the $\mathrm{n}+1$ group are obtained. Then the Newton interpolation 
formula is used to obtain the optimal stable solution of the integral equation when the regularization operator is 0 . The numerical examples show that each algorithm has high accuracy.

Shang and Han [60] proposed an effective method to solve the first kind of Fredholm integral equation. That is to say, in $[0,1]$ interval, the solution of the linear integral equation is transformed into the solution of algebraic equation system by using Galerkin method with continuous Legendre multiwavelet function as the basis function. Maleknejad et al. [61, 62] also transformed the linear integral equation into a system of linear algebraic equations using continuous Legendre wavelet as the basis function in the $[0,1]$ interval based on the Galerkin method. The convergence and error estimation of the method were analyzed by the conjugate gradient method. At the same time, the orthogonal wavelet projection method is proposed to solve the first kind of Fredholm integral equation. Based on MRA attributes, it is shown that projection methods, especially wavelets-Galerkin method (WGM), are bound to converge. Zhang [63] constructed a piecewise Legendre multiwavelet basis function on $L^{2}([0,1])$ based on the theory of multiresolution analysis of wavelet analysis and solved the integral equation by collocation method.

Babolian et al. [64] used the moment method of the wavelet basis function to reduce the dimension of linear equations, instead of converting the coefficient matrix into a sparse matrix when solving the first kind of Fredholm integral equation. The numerical examples of this method also show the effectiveness of the algorithm. Adibi and Assari [65] on the unit interval, based on the Galerkin method and Chebyshev's wavelet method, converted solving integral equation into solving algebraic equations. By using the properties of Chebyshev wavelet, the coefficient matrix of the equations is sparse. The numerical examples show the efficiency of the method. Zhang [66] proposed that the first Fredholm integral equation should be discretized into linear ill-posed equations and solved numerically by using the wavelet transform method. This method combines wavelet transform with regularization method and Schur complementary conjugate gradient method, and chooses wavelet function as a group of bases to transform the original ill-posed problem into the well-posed problem in rough subspace. The validity and feasibility of this method are verified by numerical experiments. Babaaghaie and Mesgarani [67] used a triangular wave as the basis function for the first kind of Fredholm integral equation in Holder space. The Galerkin method of triangular wavelet is used to process two-dimensional triangular wavelet. The convergence analysis of the numerical solution of two-dimensional triangular wavelet on the Holder space $C_{\alpha}([a, b])$ is given. Tahami et al. [68] present a numerical method for solving two-dimensional first kind Fredholm integral equation, which based upon twodimensional linear Legendre wavelet basis approximation.

\subsection{Multilevel iteration method}

A multiscale fast algorithm is a method for solving the integral equation developed in the 1990s. Its main idea is to construct a compact support and vanishing moment multi-scale base to discretize the integral equation so that the coefficient matrix of the algebraic equation system can be hierarchically and numerically sparse. On this basis, matrix compression is carried out to achieve the purpose of a fast solution. Chen and Xu [69] constructed a fast multiscale Petrov-Galerkin method for solving the second kind of weakly singular integral equation on the basis of discontinuous orthogonal multiscale functions. They propose a matrix compression strategy to make the compressed matrix sparse and prove that the method is stable and has an approximate optimal convergence order.

Since the equation obtained by regularization of the first kind of integral equation is a second kind of integral equation with parameters, in order to solve the equation quickly, a fast algorithm for solving the second kind of integral equation can be used. Therefore, a multi-scale fast algorithm is also applied to solve ill-posed problems. Chen et al. $[70,71]$ were the first to apply multiscale methods to the first class of ill-posed integral equations. Their main idea was to solve ill-posed integral equations by combining the Tikhonov regularization method with multiscale Galerkin method and to compress dense matrices into sparse matrices by truncation strategy. Thus, a fast algorithm could be derived.

In the process of solving the first kind of Fredholm integral equation, Li et al. [72-74] first transformed the first type of integral equation into the second type of integral equation by regularization algorithm. The integral equation was discretized by Galerkin as an equivalent equation group, and the truncated projection method was used to discretize the equation group. Then the block multi-level iterative algorithm was used to calculate the equation group, and the posterior parameter method was used to simplify it into two-step iteration. The algorithm ensures the optimal approximate solution. Considering the first kind of Fredholm integral equation with perturbation of initial data, the multi-scale Galerkin method is used to solve the first kind of Fredholm ill-conditioned integral equation, and a fast algorithm for regularization parameter selection is proposed. The multi-scale truncated projection method is used to solve the ill-conditioned integral equation with perturbation of initial data, and its computational complexity and convergence are analyzed. Rate. The modified deviation principle is used as a posteriori 
parameter selection strategy, and its approximate solution is proved to reach the optimal convergence order [75]. Yang [76] mainly studies the multiscale fast method for the first kind of Fredholm integral equation with perturbation of initial data. The first kind of Fredholm integral equation can be transformed into the second kind of Fredholm integral equation by using the regularization method. On the basis of multi-scale, a matrix compression strategy is proposed, and a suitable posterior parameter selection strategy is adopted to obtain a fast algorithm for solving the integral equation.

In [75], a numerical direct method based on hybrid Block-Pulse functions and Legendre polynomials is proposed to solve Fredholm integral equation of the first kind. Bahmanpour et al. [77] introduce Müntz wavelets by using the Müntz-Legendre polynomials on the interval $[0,1]$. Xie [78] studied the first kind of Fredholm integral equation with perturbation of original data under truncation strategy and obtained the fast solution of discrete regularization equation. Then, a posteriori regularization parameter selection method is given, and the optimal convergence rate of the approximate solution is demonstrated. A multilayer extension method for solving the first kind of Fredholm integral equation under truncation strategy is introduced, which can obtain stable solutions. The convergence rate of the solution can be improved by using iterative regularization method, and a posteriori regularization parameter selection method is given. Li et al. [79] studied the multi-layer Jacobi and Gauss-Seidel iteration methods for solving ill-posed integral equations under compression technique. The fast solution of the discrete regularization method equation is obtained by this method. A posteriori regularization parameter selection method is given, and the optimal convergence rate of the approximate solution is proved. Multilayer Jacobi and Gauss-Seidel iterative methods for solving ill-posed integral equations based on fast collocation method. The multi-layer iteration method for solving the discrete equation of the first kind of ill-posed integral equation caused by the fast collocation method is introduced, and the method for selecting the posterior regularization parameters is given.

\subsection{Smooth factor solution method}

In order to solve the first kind of Fredholm integral equation, regularization and smoothing methods were used to solve this kind of problem. These methods are based on adding some "regularity" or "smoothness" constraints, and by adjusting the corresponding parameters to find the stable solution of the equation. Strand and Westwater [80] discussed the derivation and explanation of the numerical solution of the first kind of Fredholm integral equation on the basis of a certain understanding of the nature of the solution. The covariance of prior constraint vectors, constraint vectors, and measurement errors is used. The smoothing method automatically adds an optimal maximum likelihood estimate. The problem of the optimal base vector is discussed. The accuracy of the result is estimated by the covariance matrix of the error. Empirical analysis verifies the feasibility of the algorithm. Wang [81] proposed that considering the different smoothing factors of $p$, the stable solutions of $P$ group with smoothing factors can be obtained by smoothing method, and then the stable solutions can be extrapolated by extrapolation method. Then, with Bi and Wang [82], a multi-constrained smoothing method for solving the first kind of Fredholm integral equation is proposed, and the Phillips smoothing method is improved. By introducing multiple constraints and adding different smoothing parameters to derivative constraints, the corresponding smoothing matrices are obtained. The stable solutions with multiple derivative constraints and multiple smooth parameters are obtained. Kyurkchan and Anyutin [83] simplified the well-posedness of the diffraction problem to the first kind of Fredholm integral equation with smooth kernels. The auxiliary source method and the extended boundary condition method are used to solve this kind of integral equation. The specific examples show that the algorithm is relatively stable and achieves the accuracy of the pre-estimation. Lin and $\mathrm{Xu}$ [84] studied the convergence rate of the collocation method for solving the first kind of Fredholm integral equation. By constructing an approximate interpolation neural network, it is deduced that the convergence rate of the approximate solution is only applicable to the first kind of Fredholm integral equation with continuous functions as basis functions. The convergence rate is a finite smoothing coefficient.

\subsection{Other methods}

In addition to the usual methods for solving the first kind of Fredholm integral equation, such as regularization, wavelet transform, and multi-level iteration, many scholars have studied the numerical solution of the integral equation from adaptive numerical method, discrete kernel method, optimal homotopy asymptotic method, algebraic method, Lagrange polynomial interpolation method, trust region algorithm, slow solution, collocation method, sinc collocation method, and etc [14, 85-87].

Hanson and Phillips [88] used a continuous piecewise linear function instead of the integral equation to obtain the approximate solution when solving the numerical solution of the first kind of linear Fredholm integral equation. Combining with the stable linear algebraic equations, the accuracy of the approximate solution is improved by using the improved iteration method. Koshev and Beilina 
[89] proposed an adaptive finite element method to solve the first kind of linear Fredholm integral equation. The minimum posterior error estimates and regularization solutions are obtained in the functional, and the corresponding adaptive algorithm is given. Nonlinear results are obtained based on linear bounded operators. The numerical experiments show that the efficiency of our posterior estimation is suitable for both simulation and backscattering data measured by Microtomography experiment.

Chocholat [90] solves the first kind of special Fredholm integral equation by the method of kernel basis function. The approximate solution in linear form is expressed as $u_{k}(t)=\sum w_{j} K\left(s_{j}, t\right), j=1,2, \ldots, k$. The unknown parameters $w_{j}$ and $s_{j}$ are determined by solving two linear overdetermined equation systems and a k-order polynomial equation. Test examples show that the numerical solution is quite accurate. Almousa and Ismail [91] proposed a semianalytical method, also known as the optimal homotopy (OHAM) asymptotic method, which can be used to solve the numerical solution of the first kind of linear Fredholm integral equation. Three examples are discussed to show the effectiveness of the method. The results show that this method is very effective and simple. Evans and Rahma [92] proposed a parallel computing method when studying the numerical solution of the first kind of Fredholm integral equation. The decomposition process of singular values is solved by the least square method. In the three stages of parallel computing, a suitable form is used to achieve high parallelism. The efficiency of the algorithm is verified by Loughborough Neptune system. Phillips [93] found that the solution of the first kind of Fredholm integral equation without vibration can be solved by inverse induced control smoothing of the orthogonal approximate integral equation. Twomey [94] replaces the inversion of two matrices described by Phillips with the inversion of one matrix under the same results. This solution has the advantage of allowing the orthogonal coefficient matrix to be not a square matrix.

Herr [95] proposed a statistical model for solving the numerical solution of the first kind of Fredholm integral equation. The model and related problems are essentially regression analysis. The main purpose is to give a method equivalent to Strand, Westwater problem (GLM). The research results enrich its inherent geometric properties. Zhilyakov [96] gives the appropriate approximate calculation method and verifies the practicability of the approximate calculation method by combining the empirical data with the calculation program. For the information evaluation of the first kind of Fredholm integral equation, Micheli and Viano [97] consider the case that the integral operator is the self-adjoint compact operator. In the allowable range of slight perturbation of data function. Firstly, the upper and lower bounds (as well as the measurement information) in the range of $\varepsilon$ are given, and then the results obtained by the two methods are compared by using the probability information theory, which verifies the effectiveness of the algorithm.

The algebraic method can obtain satisfactory approximate solutions when solving a class of integral equations which can be approximately expressed as algebraic polynomials. Graves and Prenter [98] applied the research of numerical filtering iteration chain to the first linear Fredholm integral equation. The validity of the study is verified by comparing the inversion results using singular value decomposition (SVD) and the direct method. Wang and $\mathrm{Wu}$ [99] proposed an algebraic solution for the numerical solution of the first kind of Fredholm integral equation. The concept of algebraic accuracy and the phase error estimate of the algebraic solution were given. The feasibility of the algorithm was verified by numerical examples. When $\mathrm{Li}$ [100] studied the approximate solution of the first kind of Fredholm integral equation, a kind of approximate approximation scheme was constructed by using the Lagrange polynomial interpolation method. The convergence of the approximate solution sequence was proved theoretically.

Trust region algorithm is a newly developed method for solving ill-posed problems. Wu [101] mainly studies the application of trust region algorithm in solving the first kind of Fredholm integral equation. The first kind of Fredholm integral equation with weak singular kernels and the first type of two-dimensional and three-dimensional Fredholm integral equation with non-singular kernels are solved by integral discrete analysis and trust region method [10]. The results are compared with those obtained by iterative Tikhonov regularization method and TSVD regularization method. The feasibility of the trust region algorithm is verified by numerical simulation and experimental results.

Based on the idea of computing all possible unknown functions, Pang [102] studied a data slow search method to solve the first kind of Fredholm integral equation. Although this method has a large amount of calculation and slow speed, it is a reliable method for an accurate solution. Moreover, with the development of the computer, the superiority of this algorithm will be more and more obvious. The feasibility and stability of the slow data search method are analyzed, and the application of the method in structural vibration parameter identification is verified by the step-by-step search method.

Maleknejad et al. [103] proposed an advanced numerical model in solving the Fredholm integral equations of the first kind by using Sinc basis functions, Which method has been shown to be a powerful numerical solution for finding accurate solutions. Didgar et al. [104] provide a new application of Taylor expansion approach for solving 
Table 1 Comparison between maximum absolute errors for Eq. 2

\begin{tabular}{llllll}
\hline Methods & $\begin{array}{l}\text { Dong } \\
\text { and Chen } \\
{[105]}\end{array}$ & $\begin{array}{l}\text { Bahman- } \\
\text { pour et al. } \\
{[77]}\end{array}$ & $\begin{array}{l}\text { Adibi and } \\
\text { Assari } \\
{[65]}\end{array}$ & $\begin{array}{l}\text { Malekne- } \\
\text { jad and } \\
\text { Saeedi- } \\
\text { poor [75] }\end{array}$ & $\begin{array}{l}\text { Male- } \\
\text { knejad } \\
\text { and }\end{array}$ \\
$\begin{array}{l}\text { Sohrabi } \\
\text { [61] }\end{array}$ \\
\hline $\begin{array}{l}\text { Max. } \\
\text { abso- } \\
\text { lute } \\
\text { error }\end{array}$ & $2.09 \mathrm{e}-5$ & $4.21 \mathrm{e}-5$ & $8.92 \mathrm{e}-4$ & $9.01 \mathrm{e}-4$ & $8.54 \mathrm{e}-4$ \\
\hline
\end{tabular}

first kind Fredholm integral equations, wchich is based on employing the $v$ th-degree Taylor polynomial of unknown function at an arbitrary point.

\section{Numerical examples}

In this section, numerical results are given to show the efficiency and accuracy of some representative algorithms. Consider the following Fredholm integral equation:

$\int_{0}^{1} e^{s t} f(s) d s=\frac{e^{t+1}-1}{t+1}$

The exact solution to this equation is $f(t)=e^{t}$. Table 1 shows the maximum absolute errors about some representative algorithms. As can be seen from Table 1, with the gradual improvement of the algorithm, the relative error is getting smaller and smaller. It shows that the solution of this problem is more and more mature.

\section{Conclusions and prospects}

In this paper, we provide an overview of numerical methods for first kind Fredholm integral equation. The numerical solution of the first kind of Fredholm integral equation promotes the progress of science and technology to a great extent. Many scholars not only solve some engineering and technical problems but also play an important role in image processing and medical imaging. From the point of view of the current methods for solving the first kind of Fredholm integral equation, the main method is to use regularization algorithm and adopt different regularization operators to solve different integral equations; or to use the wavelet method to solve, give a set of wavelet bases as the basis function to approximate the integral equation, and solve the integral equation by improving the basis function or improving the iterative algorithm. In addition, multi-scale iteration method, Lagrange polynomial interpolation method, algebraic solution method, statistical method, orthogonal inversion method, homotopy asymptotic method and so on can be applied to specific equations. These methods are all approximated from different angles. It is not only difficult to select approximation function, but also difficult to solve the numerical solution of approximation function. We hope to be able to give a simple discrete formula (trapezoid, compound trapezoid, Simpson, compound Simpson, etc.) to discretize the integral equation into linear algebraic equations, but for large linear equations, the effect of the general iterative algorithm is not obvious.

The modern intelligent optimization algorithm is a kind of calculation method with self-adaptive environment ability, which is developed by using the principles and mechanisms of natural phenomena or organisms in nature for reference. It mainly includes a genetic algorithm, neural network optimization, particle swarm optimization, and simulated annealing algorithm. Annealing, etc. These algorithms are constructed by revealing and simulating natural phenomena and engineering practice, and synthetically utilizing physics, biological evolution, artificial intelligence, and neuroscience. They are also called heuristic algorithms. This kind of algorithm provides a new way of thinking for engineering and technical problems because of its randomness, rapidity, and efficiency in the process of optimization. If the intelligent algorithm is used to solve the discrete algebraic equations of the integral equation, the ideal result may be obtained.

Acknowledgements This research was supported by the Shenzhen Research Council (Grant Nos. JCYJ20160406161948211, JCYJ20160226201453085, JSGG20150331152017052 and JCYJ20160531194006833), by the National Natural Science Foundation of China (Grant Nos. 61672183, 61272366, 61672444 and 41004052), by Science and Technology Planning Project of Guanddong Province (Grant Nos. 2016B090918047 and 2017A030313280).

\section{Compliance with ethical standards}

Conflict of interest The authors declare that they have no conflict of interests.

\section{References}

1. Bakushinskii $A B$ (1965) A numerical method for solving Fredholm integral equations of the 1st kind. USSR Comput Math Math Phys 5(4):226-233

2. Denisov AM (1971) Approximation of quasi-solutions of Fredholm's equation of the first kind with a kernel of special form. USSR Comput Math Math Phys 11(5):269-276

3. Denisov AM (1972) Approximation of the quasi-solutions of a Fredholm integral equation of the first kind of a special form. USSR Comput Math Math Phys 12(6):244-248

4. Denisov AM (1974) On the order of approximation when solving a Fredholm equation of the first kind with a kernel of special type. USSR Comput Math Math Phys 13(1):255-260 
5. Groetsch CW (1990) Convergence analysis of a regularized degenerate kernel method for Fredholm integral equations of the first kind. Integral Equ Oper Theory 13(1):67-75

6. Yuan D, Lu S, Li D, Zhang X (2019) Graph refining via iterative regularization framework. SN Appl Sci 1(387):1-10

7. Rajan MP (2004) A modified convergence analysis for solving Fredholm integral equations of the first kind. Integral Equ Oper Theory 49(4):511-516

8. Maleknejad K, Mollapourasl R, Nouri K (2006) Convergence of numerical solution of the Fredholm integral equation of the first kind with degenerate kernel. Appl Math Comput 181(2):1000-1007

9. Yuan D, Zhang X, Liu J, Li D (2019) A multiple feature fused model for visual object tracking via correlation filters. Multimedia Tools Appl. https://doi.org/10.1007/s11042-019-07828 $-2$

10. Hamedzadeh D, Babolian E (2018) A computational method for solving weakly singular Fredholm integral equation in reproducing kernel spaces. Iran J Numer Anal Optim 8(1):1-17

11. Yuan D, Lu X, Li D, Liang Y, Zhang X (2019) Particle filter redetection for visual tracking via correlation filters. Multimedia Tools Appl 78(11):14277-14301

12. Yang $\mathrm{H}$, Gong $\mathrm{Z}$ (2019) III-posedness for fuzzy Fredholm integral equations of the first kind and regularization methods. Fuzzy Sets Syst 358:132-149

13. Ou W, Yuan D, Li D, Liu B, Wu Z (2017) Patch-based visual tracking with online representative sample selection. J Electron Imaging 26(3):033006

14. Hafez R, Doha E, Bhrawy A, Baleanu D (2017) Numerical solutions of two-dimensional mixed Volterra-Fredholm integral equations via bernoulli collocation method. Roman J Phys 62(3):1-11

15. Wei $P$ (2007) Integral equations and their numerical methods. Metallurgical Industry Press, Beijing, pp 70-89

16. Lu K, Zhong S (2008) Integral equation theory. Wuhan University Press, Wuhan, pp 155-162

17. Yun $T$ (1989) Representation theorem and one-iteration theorem for Fredholm integral equation of the first kind $a x \doteq y$. Appl Math Mech 07:593-597

18. Li C, Zhao B (1998) Reliability of the numerical solution to the Fredholm integral equation of the first kind. J Appl Sci 3(3):285-290

19. Hong D, Cui M (2006) Representation of the exact solution and a stability analysis on the Fredholm integral equation of the first kind in reproducing kernel space. Appl Math Comput 182(2):1608-1614

20. Zhang Q, Wang L (2009) Existence of positive solutions for a class of Fredholm integral equations. J Jilin Univ (Science Edition) 47(3):481-486

21. Liu M (2011) Error estimate on direct discretization of Fredholm integral equation of the first kind. J Nat Sci Heilongjiang Univ 28(1):17-21

22. Han H, Li Y, Yin D, Chen Z (2015) The necessary and suficient condition for the existence and uniqueness of a system of Fredholm integral equations of the first kind. Sci China Math 45(08):1231-1248

23. He R, Zhang L (2018) Numerical methods for Fredholm integral equations of the first kind. In: International conference on physics, computing and mathematical modeling, pp 133-138

24. Lin F, Yang S (2017) A two-stage method for piecewise-constant solution for Fredholm integral equations of the first kind. Mathematics 5(2):1-16

25. Shoukralla E, Kamel M, Markos M (2018) A new computational method for solving weakly singular Fredholm integral equations of the first kind. In: 2018 13th international conference on computer engineering and systems, pp 202-207
26. Priyadarshi G, Kumar BR (2018) On the existence of approximate solution of Fredholm integral equation of the first kind by band-limited scaling function. Differ Equ Dyn Syst. https ://doi.org/10.1007/s12591-018-0416-8

27. Lee JW, Prenter PM (1978) An analysis of the numerical solution of Fredholm integral equations of the first kind. Numerische Mathematik 30(1):1-23

28. Khromov AA, Khromova GV (2009) Finding approximations of continuous solutions to first-kind equations. Comput Math Math Phys 49(2):217-223

29. Perminov VD (1977) Numerical algorithm for the solution of linear two-dimensional integral equations of the first kind. J Eng Phys 33(6):1493-1496

30. You Y, Miao G (1998) On the regularization method of the first kind of Fredholm integral equation with a complex kernel and its application. Appl Math Mech 19(1):75-83

31. Pereverzev SV, Semenova EV, Tkachenko P (2018) Regularized quadrature methods for Fredholm integral equations of the first kind. In: Contemporary computational mathematics-a celebration of the 80th birthday of Ian Sloan. pp. 1017-1034

32. Tikhonov AN, Goncharsky AV, Stepanov VV, Yagola AG (1995) Numerical methods for the solution of III-posed problems. Kluwer, Dordrecht

33. Tanana VP, Vishnyakov EY, Sidikova Al (2016) About an approximate solution to the Fredholm integral equation of the first kind by the residual method. Numer Anal Appl 9(1):74-81

34. Caldwell J (1994) Numerical study of Fredholm integral equations. Int J Math Educ Sci Technol 25(6):831-836

35. Pereverzeva GA (1999) Projection methods for the solution of Fredholm integral equations of the first kind with $(\phi, \beta)$-differentiable kernels and random errors. Ukr Math J 51(5):793-798

36. Dobner HJ (2002) Kernel-splitting technique for enclosing the solution of Fredholm equations of the first kind. Reliab Comput 8(6):469-479

37. Li G, Liu Y (2005) A new regularizing algorithm for solving the first kind of Fredholm integral equations. J Math Res Appl 25(2):204-210

38. Li G, Zhang R, Pan Y, Bian Q (2003) An optimal regularization strategy for solving first kind of Fredholm integral equations. J Shandong Univ Technol (Sci Technol) 17(1):72-75

39. Li G, Yu J (2003) An optimal regularization strategy for solving first kind of Fredholm integral equations. J Shandong Univ Technol (Sci Technol) 17(2):5-8

40. Maleknejad K, Aghazadeh N, Mollapourasl R (2006) Numerical solution of Fredholm integral equation of the first kind with collocation method and estimation of error bound. Appl Math Comput 179(1):352-359

41. Dan B, Shklyar BZ (2008) Existence and approximation of solutions for Fredholm equations of the first kind with applications to a linear moment problem. Optim Methods Softw 23(1):21-37

42. Nair MT, Pereverzev SV (2007) Regularized collocation method for Fredholm integral equations of the first kind. J Complex 23(4):454-467

43. Maleknejad K, Saeedipoor E, Dehbozorgi R (2016) Legendre wavelets direct method for the numerical solution of Fredholm integral equation of the first kind. In: Proceedings of the world congress on engineering, vol 1

44. Zeng H, Lv X, Luo W (2015) A collocation method for the first kind of weakly singular Fredholm integral equations. J Neijiang Normal Univ 10:10-13

45. Rajan MP (2010) A parameter choice strategy for the regularized approximation of Fredholm integral equations of the first kind. Int J Comput Math 87:2612-2622 
46. Wazwaz AM (2011) The regularization method for Fredholm integral equations of the first kind. Comput Math Appl 61(10):2981-2986

47. Micheli ED, Viano GA (2012) Fredholm integral equations of the first kind and topological information theory. Integral Equ Oper Theory 73(4):553-571

48. Yin $F(2013)$ The discreted regularization method of solving the first kind Fredholm integral equation. Sci Technol Vis 31:151-152

49. Yang P, Wu J, Wu K (2013) Regularization method for first kind Fredholm equation with infinite range. J Beijing Univ Chem Technol (Nat Sci Edn) 40(S1):117-121

50. Min T, Zhao M (2012) Fredholm integral equations for solving the first class of three-dimensional gmres method. Acta Anal Funct Appl 14(3):234-238

51. Min T, Hu G, Yan L (2014) A numerical solution of weakly singular Fredholm integral equation of first kind. Acta Anal Funct Appl 16(2):167-171

52. Lin FR, Yang SW (2014) A weighted h1 seminorm regularization method for Fredholm integral equations of the first kind. Int J Comput Math 91(5):1012-1029

53. Wu H (2008) The study of numerical algorithms for fredholm integral equations of the first kind. PhD thesis, Xi'an University of Technology

54. Zhang X (2013) The regularization for the first kind of Fredholm integral equation. PhD thesis, University of Electronic Science and Technology of China

55. Mao H (2010) The Tikhonov regularization method for Fredholm equation of the first kind with applications. PhD thesis, Shanghai Jiaotong University

56. Yousefi S, Banifatemi A (2006) Numerical solution of Fredholm integral equations by using cas wavelets. Appl Math Comput 183(1):458-463

57. Wang $\mathrm{Y}$, Han $\mathrm{H}$ (2009) Cas wavelet method for Fredholm integral equation of the first kind. Sichuan Ordnance J 30(2):127-128

58. Zhu L, Han $\mathrm{H}$ (2009) Solution to Fredholm integral equation of the first kind by using legendre multi-wavelets. Sichuan Ordnance J 30(2):127-128

59. Zhang J, Han H, Pan X (2010) Wavelet-regularization method and extrapolation for solving Fredholm integral equations of the first kind. Sci Technol Eng 10(1):17-20

60. Shang X, Han D (2007) Numerical solution of Fredholm integral equations of the first kind by using linear legendre multiwavelets. Appl Math Comput 191(2):440-444

61. Maleknejad K, Sohrabi S (2007) Numerical solution of Fredholm integral equations of the first kind by using legendre wavelets. Appl Math Comput 186(1):836-843

62. Maleknejad K, Lotfi T, Mahdiani K (2007) Numerical solution of first kind Fredholm integral equations with wavelets-galerkin method (wgm) and wavelets precondition. Appl Math Comput 186(1):794-800

63. Zhang J (2013) Legendre multi-wavelets adaptive method for first category Fredholm integral equations. Comput Digit Eng 41(6):874-875

64. Babolian E, Lotfi T, Paripour M (2007) Wavelet moment method for solving Fredholm integral equations of the first kind. Appl Math Comput 186(2):1467-1471

65. Adibi H, Assari P (2010) Chebyshev wavelet method for numerical solution of Fredholm integral equations of the first kind. Math Problems Eng. https://doi.org/10.1155/2010/138408

66. Zhang H (2011) Application of wavelet transform in solving rust kind of Fredholm integral equation. JTangshan Coll 24(3):9-11

67. Babaaghaie A, Mesgarani H (2012) Numerical solution of Fredholm integral equations of first kind by two-dimensional trigonometric wavelets in holder space $c \alpha([a, b])$. Comput Math Math Phys 52(4):601-614
68. Tahami M, Hemmat AA, Yousefi SA (2016) Numerical solution of two-dimensional first kind Fredholm integral equations by using linear legendre wavelet. Int J Wavelets Multiresolut Inf Process 14(01):1650004

69. Chen Z, Xu Y (1998) The Petrov-Galerkin and iterated PetrovGalerkin method for second-kind integral equations. SIAM J Numer Anal 35(1):406-434

70. Chen $Z, X u Y$, Yang $H$ (2006) A multilevel augmentation method for solving ill-posed operator equations. Inverse Problems 22(1):155-174

71. Chen Z, Xu Y, Yang H (2008) Fast collocation methods for solving ill-posed integral equations of the first kind. Inverse Problems 24(6):065007

72. Li F, Yang S, Luo X, Peng Y (2013) Multilevel iterative algorithm for solving Fredholm integral equation of the first kind. Math Numer Sin 35(3):225-238

73. Luo X, Xie Z, Fan L, Li F (2015) A multiscale galerkin method for Fredholm integral equations of the first kind with perturbed initial data based on truncated method. Adv Math (China) 44(2):305-319

74. Yang X, Luo X, Yang S, Li F (2016) A fast multiscale method for the Fredholm integral equation with nonexactly given input data via modified discrepancy principle. Numer Math J Chin Univ 37(2):111-130

75. Maleknejad K, Saeedipoor E (2017) An efficient method based on hybrid functions for Fredholm integral equation of the first kind with convergence analysis. Appl Math Comput 304:93-102

76. Yang X (2014) Solving the Fredholm integral equation of the first kind with perturbed initial data based on truncation strategy. PhD thesis, Gannan Normal University

77. Bahmanpour M, Kajani MT, Maleki M (2019) Solving Fredholm integral equations of the first kind using Muntz wavelets. Appl Numer Math 143:159-171

78. Xie F (2014) A multiscale multiscale method for Fredholm integral equations of the first kind based on truncated method. PhD thesis, Gannan Normal University

79. Luo X, Hu W, Xiong L, Li F (2015) Multilevel jacobi iteration methods for solving ill-posed integral equations. J Gannan Norm Univ 23(5):3-8

80. Strand ON, Westwater ER (1968) Statistical estimation of the numerical solution of a Fredholm integral equation of the first kind. JACM 31(15):100-114

81. Wang D (2006) A stable method for calculating Fredholm integral equation of first kind. J Tongji Univ (Nat Sci) 34(10):1414-1416

82. Bi B, Wang D (2008) Multi-constraint smooth method for solving Fredholm integral equation of first kind. J Harbin Inst Technol 40(3):440-442

83. Kyurkchan AG, Anyutin AP (2006) The well-posedness of the formulation of diffraction problems reduced to Fredholm integral equations of the first kind with a smooth kernel. J Commun Technol Electron 51(1):48-51

84. Lin S, Xu Z (2012) A convergence rate for approximate solutions of Fredholm integral equations of the first kind. Positivity 16(4):641-652

85. Maleknejad K, Ostadi A (2017) Using sinc-collocation method for solving weakly singular Fredholm integral equations of the first kind. Appl Anal 96(4):702-713

86. Karimi S, Jozi M (2015) A new iterative method for solving linear Fredholm integral equations using the least squares method. Appl Math Comput 250:744-758

87. Michel V, Orzlowski S (2016) On the null space of a class of Fredholm integral equations of the first kind. J Inverse IIIPosed Problems 24(6):687-710 
88. Hanson RJ, Phillips JL (1975) An adaptive numerical method for solving linear Fredholm integral equations of the first kind. Numer Math 24(4):291-307

89. Koshev N, Beilina L (2013) An adaptive finite element method for Fredholm integral equations of the first kind and its verification on experimental data. Cent Eur J Math 11(8):1489-1509

90. Chocholaty P (1993) A numerical treatment to the solution of certain first kind Fredholm integral equations. Calcolo 30(3):191-202

91. Almousa M, Ismail A (2013) Optimal homotopy asymptotic method for solving the linear Fredholm integral equations of the first kind. Abstr Appl Anal 2013(9-12):432-445

92. Evans DJ, Rahma AMS (1989) The numerical solution of Fredholm integral equations on parallel computers. Parallel Comput 10(2):193-205

93. Phillips DL (1962) A technique for the numerical solution of certain integral equations of the first kind. JACM 9(1):84-97

94. Twomey S (1963) On the numerical solution of Fredholm integral equations of the first kind by the inversion of the linear system produced by quadrature. JACM 10(1):97-101

95. Herr DG (1974) On a statistical model of strand and westwater for the numerical solution of a Fredholm integral equation of the first kind. JACM 21(1):1-5

96. Zhilyakov EG (2003) On the computation of approximate solutions of Fredholm integral equations of the first kind with the use of empirical data. Differ Equ 39(7):968-972

97. Micheli ED, Viano GA (2005) Metric and probabilistic information associated with Fredholm integral equations of the first kind. J Integral Equ Appl 14(3):283-309
98. Graves J, Prenter PM (1978) Numerical iterative filters applied to first kind Fredholm integral equations. Numer Math 30(3):281-299

99. Wang D, Wu R (1991) The algebrac solution of the Fredholm integral equation of the first kind. J Harbin Inst Technol 2:1-7

100. Li M (1985) The numerical solution of the first kind Fredholm integral equations. J Appl Sci 3(4):306-312

101. Wu M (2010) The application of trust region algorithm in solving the equation of the first kind. PhD thesis, Xi'an University of Technology

102. Pang A (2013) A slow method for solving Fredholm integral equations. PhD thesis, Harbin Institute of Technology

103. Maleknejad K, Rostami Y, Kalalagh HS (2016) Numerical solution for first kind Fredholm integral equations by using sinc collocation method. Int J Appl Phys Math 6(3):120

104. Didgar M, Vahidi A, Biazar J (2019) Application of taylor expansion for Fredholm integral equations of the first kind. J Math 51(5):1-14

105. Dong Y, Chen $L$ (2019) Numerical research of Fredholm integral equations of the first kind based on the legendre wavelets. Math Pract Theory 49(1):241-246

Publisher's Note Springer Nature remains neutral with regard to jurisdictional claims in published maps and institutional affiliations. 